\title{
Grain Changing Simulation of the Copper Sheet Indented by a Conical Diamond for a micro hole forming
}

\author{
SHI Guang Feng ${ }^{a}$, CAO Kong Yu \\ Changchun University of Science and Technology, School of Mechanical and Electrical Engineering, \\ Changchun China \\ ashiguangfeng@cust.edu.cn, b15714402142@qq.com
}

\begin{abstract}
Keywords: Grain changing, Conical diamond, Indentation, Hole forming, Deform 3D
Abstract. The method of micro hole forming indented by a conical diamond into a metal sheet based on glass substrate is an innovation. In order to solve the cracks problem of the hole edge during the indention experiment at present, the changing simulation of grain size and effective stress of the copper sheet material indented by a conical diamond is researched using the Deform 3D software with the point tracking function in this paper to reveal the mechanics changing regularity. And the mechanics of micro structure reason is analyzed with some solving method provided. The work in this paper can guide the further research with the visual simulation results without experiment cost.
\end{abstract}

\section{Introduction}

With the progress of science technology and industrial production, the micro-hole processing and application are more and more widely. At present, there are more than 50 kinds of micro-hole machining technologies, and many predecessors have contributed a lot in this field at home and abroad. But the machining of micro-hole with large depth to diameter ratio is still a problem in manufacturing industry up to present, and the ratio up to1000 of micro hole machining is a world-class challenge ${ }^{[1]}$.

The method of micro hole forming indented by a conical diamond into a metal sheet based on a harder substrate has be provided and researched, and the simulation and experiment results both revealed the feasibility of this method ${ }^{[2]}$. The micro conical hole in the copper sheet can be formed surprisingly, even a ultra micro hole aim at the metal sheet bottom can be pursued with a better machining process. But the micro cracks along the hole at the bottom that are not good for the workpiece application were observed, which may be caused by the micro structure change of the copper sheet material during indentation ${ }^{[3]}$.

So the grain changing during the conical diamond indenting into the copper sheet based on a glass blank is simulated by using the Deform 3D software ${ }^{[4]}$ in this paper, and the relative characteristics are analyzed to try to yield new ideas for solving the problem of the hole edge cracks.

\section{FEA simulation model of indentation for hole forming}

The FEA simulation model of indentation for hole forming in Literature [2] can be used in this paper directly as shown in Fig.1. The material lateral plastic flow can be seen in Fig.2 with the micro hole forming at the sheet bottom as shown in Fig.3. It indicates that hard substrates are more conducive to get the micro-hole by the lateral metal flow.

Then the simulated result can be imported into the "Microstructure" module of the post processor in Deform 3D software for the grain changing analysis, and a primary set is 50 micrometer for the initial grain size. Before the solving, the coordinate origin is set on the center of the top surface of the copper sheet model and a series of tracking points are set in the coordinate of the copper sheet model as shown in Fig.4. 


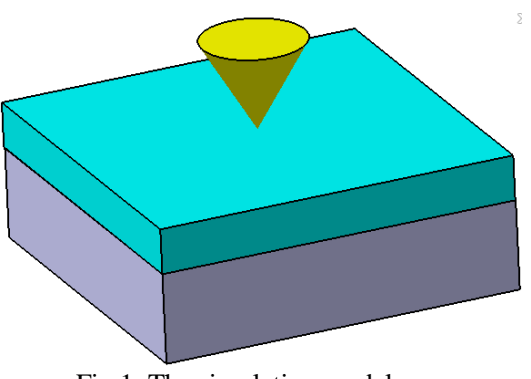

Fig.1. The simulation model

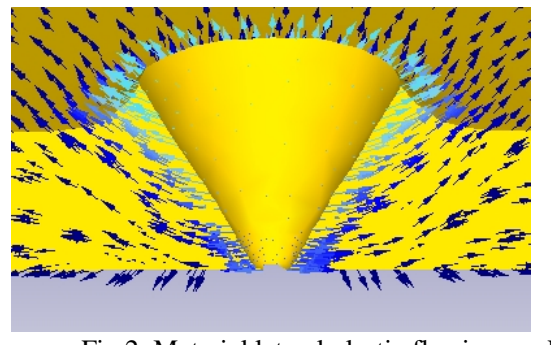

Fig.2 Material lateral plastic flowing

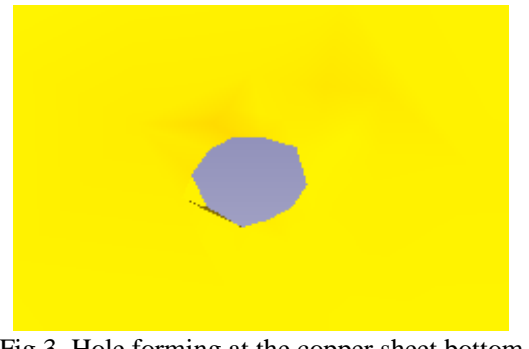

Fig. 3 Hole forming at the copper sheet bottom

The tracking points are selected as: $1(0,0,-0.3), 2(0,0.0025,-0.3), 3(0,0.005,-0.3), 4(0,0.01,-0.3)$, $5(0,0,-0.4), 6(0,0.0025,-0.4), 7(0,0.005,-0.4), 8(0,0.01,-0.3), 9(0,0,-0.45), 10(0,0.0025,-0.45)$, $11(0,0.005,-0.45), \quad 12(0,0.01,-0.45), \quad 13(0,0,-0.475), \quad 14(0,0.0025,-0.475), \quad 15(0,0.005,-0.475)$, $16(0,0.01,-0.475), 17(0,0,-0.5), 18(0,0.0025,-0.5), 19(0,0.005,-0.5), 20(0,0.01,-0.5)$. But for a simplified analysis, the point number $1,5,9,13,17$ set for P1, P2, P3, P4, P5 are chosen for analysis in detail. Then the computation process can be started with the analysis below to reveal the yielding reason of the hole-edge micro cracks after indentation experiment as shown in Fig.5.

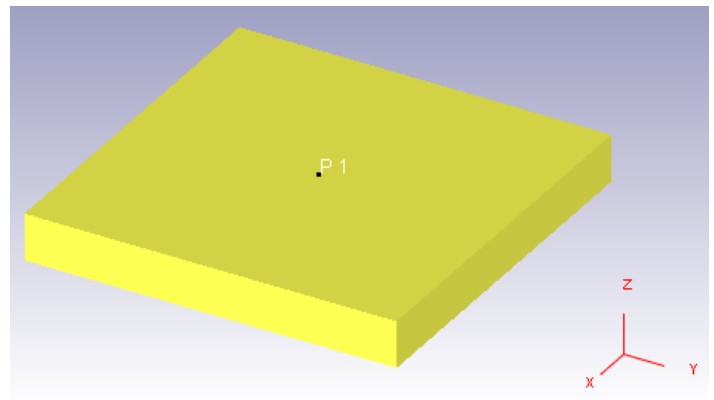

Fig.4. The coordinate

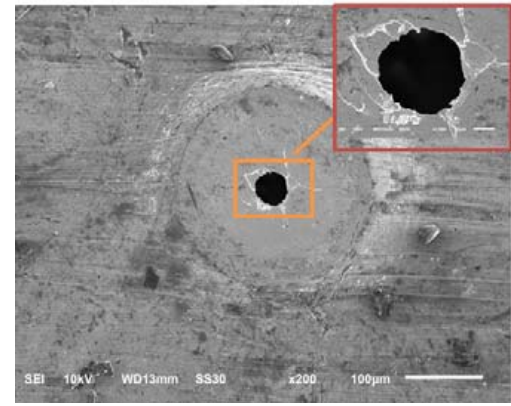

Fig.5 The SEM test result of the hole-edge micro cracks

\section{Grain size and effective stress changing analysis}

After solved, the post processor information can be treated. When the indentation depth is $0.294 \mathrm{~mm}$, the $\mathrm{P} 1$ point is just below the conical diamond tip, while the tracking results of the five points can be seen in Fig.6. The average grain sizes of the tracking points all become smaller than the initial setting value, and the $\mathrm{P} 1$ and $\mathrm{P} 2$ values are larger than the values of $\mathrm{P} 3, \mathrm{P} 4$ and $\mathrm{P} 5$ points.
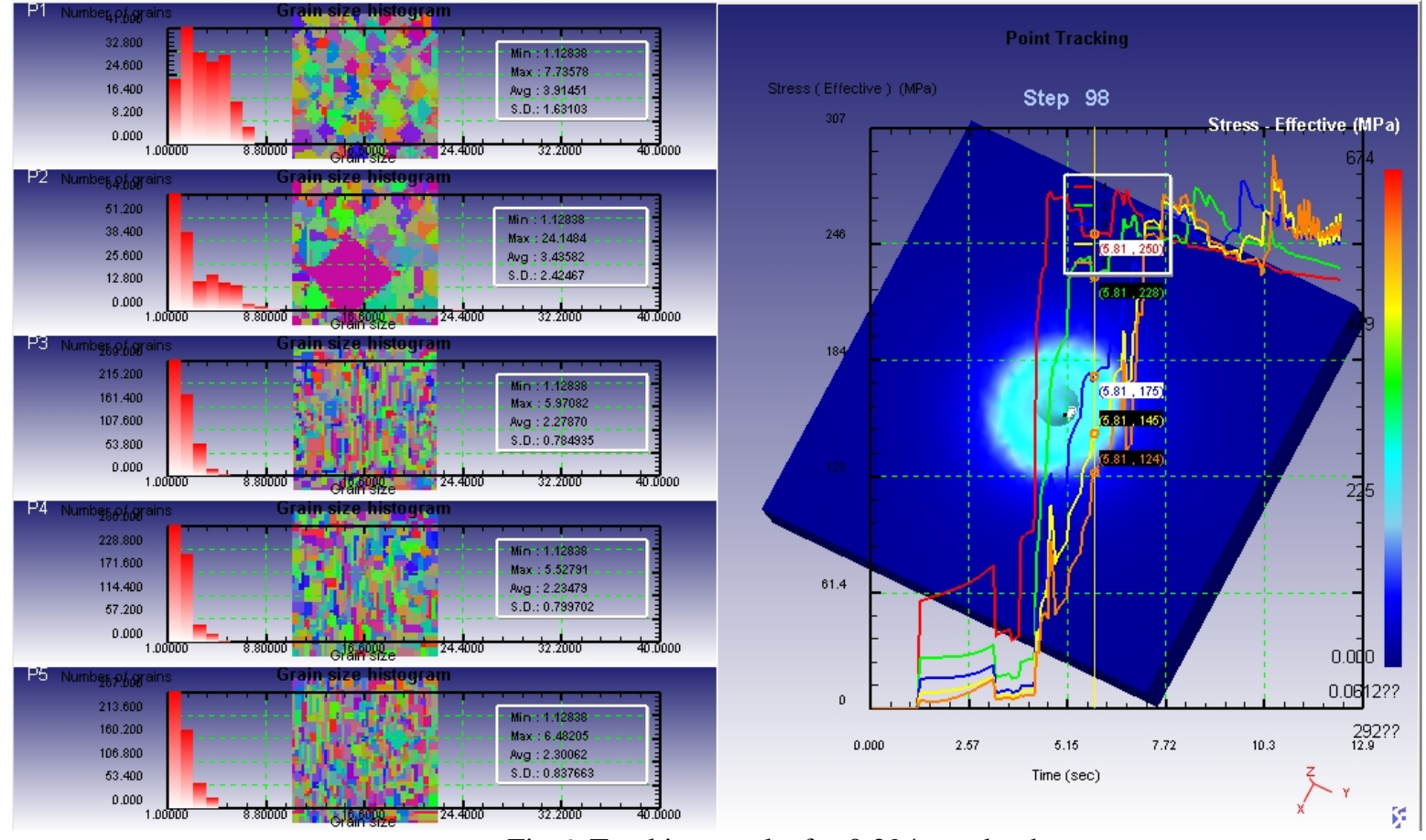

Fig.6. Tracking results for $0.294 \mathrm{~mm}$ depth 
The indentation hole forming process is a shear slip mechanics. With the conical diamond indenter go through into the copper sheet, the copper material will be divided to flow around. This forming process will cause the copper grain elongated, and nearer the indenter longer the grain. But the grain size values of P3, P4 and P5 points become much smaller than the former tow points. It indicates that the indentation pressure will cause the grain refinement ${ }^{[5]}$ far below the indenter tip, because the results in Fig.7 have different conclusions when the indentation depth increases until the bottom hole is formed. The grain sizes all become larger than the ones in Fig.6. So it shows that the former grain refinement effect will be destroyed by the increasing indentation until the bottom hole formed with a higher max effective stress $745 \mathrm{MPa}$ than the former max $674 \mathrm{MPa}$.

Furthermore, the increased hardness will block the lateral plastic flowing in the copper material, so the force action will cause the final formed hole with micro cracks yielding around the edge to emit the residential energy. Of course, the final grain sizes of the copper material all become smaller the initial size velue $5 \mu \mathrm{m}$ due to the indentaion action.

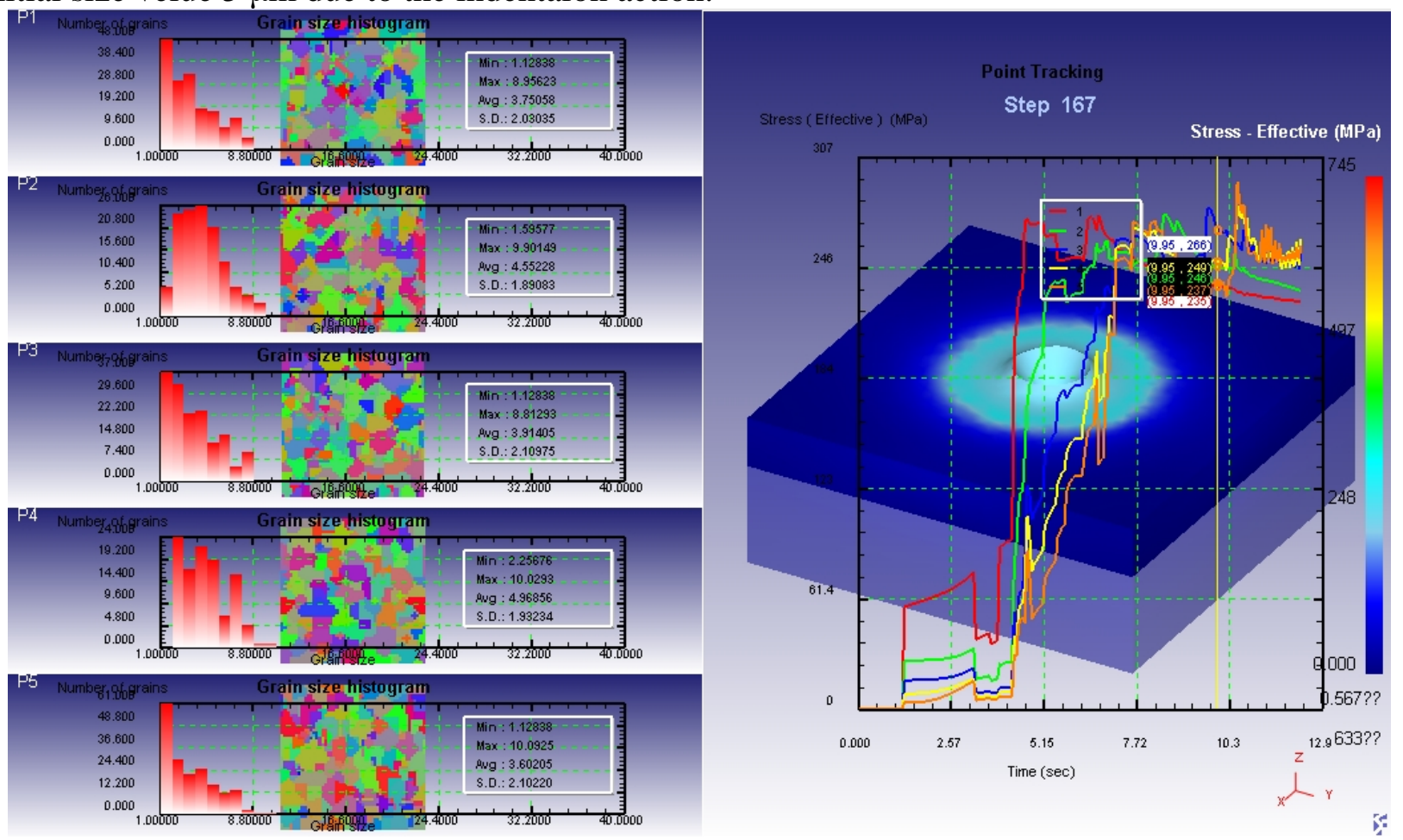

Fig.7. Tracking results for $0.5 \mathrm{~mm}$ depth

\section{Solving method discussion}

The indentation force action is a cross scale mechanics function from macro to micro, because the nose radius of the diamond indenter tip is as the same rank as the grain size. The distribution random the grain in the copper sheet material will cause the final hole forming mechanics complicated. Of course the diamond indenter can be made of natural single crystal diamond to replace the PCD material used in the present study, because the indenter of the single crystal diamond can be ripped with a sharper tip up to several dozens of nanometers. But more indentation depth will be realized to form a larger hole for certain requirement with the risk to destroy the glass substrate. And the hole forming spring back is another problem for a ultra micro hole realization.

Maybe the assistant machining method can be used here, such as aided with heat or laser ${ }^{[6]}$. Base on the temperature effect on the material constitutive relation, the gain size can be changed with certain temperature control which can be realized by the aided heat or laser. The further research will be carried out in future. 


\section{Conclusions}

In order to solve the cracks problem of the hole edge during the indention experiment at present, the changing simulation of grain size and effective stress of the copper sheet material indented by a conical diamond is researched using the Deform 3D software with the point tracking function. The mechanics changing regularity shows that the grain size below the indenter will be refined when the diamond indenter reaches the middle of the copper sheet though the grain size will be elongated around the indenter in the copper material. And the high indentation pressure will cause an increased effective stress to harden the copper material below the indenter tip, which will destroy the grain refinement formed by the former action. The cross scale mechanics may be the main reason of the micro cracks occur around the hole edge. Finally, some solving methods are provided, such as aided by heat or laser. The work in this paper can guide the further research with the visual simulation results without experiment cost, although the final result must be tested by experiment.

Some further investigations will be carried out in future so that this innovative method for hole forming can be improved.

\section{Acknowledgements}

This work was financially supported by the Jilin province science and technology development projects (No. 20150204059GX, No. 20170101124JC).

\section{References}

[1] Sen M, Shan H S. A review of electrochemical macro-to micro-hole drilling processes[J]. International Journal of Machine Tools and Manufacture, 2005, 45(2): 137-152.

[2] S. GuangFeng, J.Nana, Li. Jing, S. Guoquan. "A pore forming method on copper sheet bottom with glass substrate by diamond tip indenting[M]." Material Engineering and Mechanical Engineering: Proceedings of Material Engineering and Mechanical Engineering (MEES2015). 2016. 956-963.

[3] Yang B, Vehoff H. Dependence of nanohardness upon indentation size and grain size-a local examination of the interaction between dislocations and grain boundaries[J]. Acta Materialia, 2007, 55(3): 849-856.

[4] Fratini L, Buffa G, Palmeri D. Using a neural network for predicting the average grain size in friction stir welding processes[J]. Computers \& Structures, 2009, 87(17): 1166-1174.

[5] Jia D, Ramesh K T, Ma E. Effects of nanocrystalline and ultrafine grain sizes on constitutive behavior and shear bands in iron[J]. Acta materialia, 2003, 51(12): 3495-3509.

[6] Vrancken B, Thijs L, Kruth J P, et al. Heat treatment of Ti6Al4V produced by Selective Laser Melting: Microstructure and mechanical properties[J]. Journal of Alloys and Compounds, 2012, 541: $177-185$. 